Universitas Banten Jaya

\title{
CODE SWITCHING USED BY ENGLISH TEACHER AT ISLAM TIRTAYASA JUNIOR HIGH SCHOOL (EIGHT GRADE)
}

(Case Study at Eight Grade of Islam Tirtayasa Junior High School)

\author{
Lia Amalia', Riki Gunawan ${ }^{2}$ \\ liaamalia@unbaja.ac.id ${ }^{1}$, rikigunawan048@ gmail.com²
}

\begin{abstract}
Objective of this research is to explain the use of code switching by English teachers in classroom during learning activities. This research uses qualitative approach with a case study design, the research subject is an English teacher at Islam Tirtayasa Junior High School. Collecting data in this study using data display, data reduction and drawing conclusions. The focus of this research is the use of code switching by English teachers in online classroom during the learning process and the sub focus of the research is the types of code switching used by the English teacher and the reasons for using code switching. The result of this research is to find the type of code switching usage and the reasons for using code switching by English teachers in online classroom during the learning process. The conclusion is there are three types of code switching used by English teachers during the learning process in the classroom. Namely: First, inter sentential code switching, which is 64 times. Second, Intra-sentential code switching 31 times. Third, Tag switching 10 times. Then some reasons why English teachers use code switching, namely: First, Talking about Particular topic. Second is Interjection. Third is Repitition for Clarification. Fourth is Expressing for Group Identity.
\end{abstract}

Keyword: Code Switching, English Teacher, Islam Tirtayasa Junior High School.

\section{INTRODUCTION}

Language is the ability that people have to communicate with other people using signs, such as words and movements. The scientific study of language is calling linguistics. Study of language is very important, because language is a tool of communication. In everyday life we always using the language for communicate. Indonesian society use Indonesian language or foreign language in a capital city but sometime they are use local language in 
Journal of English Language Teaching and Literature (JELTL)

Universitas Banten Jaya

their home or their village as their first language.

Furthermore, they are also required foreign languages such as English, Arabic, Chinese, and other language to communicate with foreign people. The important of languages becomes essential to be known and learn, and also most of Indonesian people are bilingual. People who mastery languages, sometime to switch from are language into another language because of the repertoire in their minds. Therefore, this phenomenon can be called "Code-Switching in Sociolinguistics".

J. Holmes (2013, P.1) said "Sociolinguists study relationship between language and society. They are interested in explaining why we speak different social contexts, and they are concerned with identifying the social functions of language and to convey the meaning". In the other hand, Code-Switching is used of two language or multiple languages in the words, phrases, and sentences. It is support by Hudson (2012, P.51) "Code-Switching, which is the inevitable consequences of more than one language choices, they use them depend on the situation" but it also occurs in the classroom during the process of teaching and learning to explaining the material.
Before the writer found the problem about mastery of language, the writer was observed and gives some question to Mrs. Septi Hartati as English teacher in Islam Tirtayasa Junior High School at January $15^{\text {th }} 2020$. From observation the writer can found the problem such as sometime they have difficulty to understand the material if the teacher using full English language in their classroom and the students has limit vocabularies. From this problem, the teacher can use code switching in order the students can understand the material that explain by the teacher. In the other word, code switching is also more important for junior high school, because they have limited vocabularies. It is the way to improve their comprehension about the material.

Islam Tirtayasa Junior High School is formal education but the students required English language when English subjects process. In the other hand, their background education is different, exactly in mastery the language. The recruitment of students does not depend on the interest of the students. When the students back to school, they use Indonesian language and English language in classroom. So, this is school know and aware about the important using foreign language and will applied bilingual class. 
Universitas Banten Jaya

Based on explanation above, the writer is interest to analyze code switching used by English teacher when learning process. Specifics on to find out the types of code switching and what the reason of code switching used by English teacher.

\section{METHOD}

In this research, the writer uses the qualitative approach and case study methods to analyses code switching used by English teacher in Eight Grade at Islam Tirtayasa Junior High School.

The writer chooses qualitative approach for this research to express an opinion or community feedback, detail is found the precise particulars of such matters as people's understanding and interactions. Because qualitative is primarily exploratory research, it is use to gain an understanding of underlying reason, opinion and motivation. Qualitative also use to uncover trends in thought, opinion and dive deeper into problem. Therefore, the writers also focus on chase study because relates to social life behaviors and the writer focus on one person from the activities or process. In this research, the writer used several instruments to collecting data is documentation, observation and interview. In analyzed the data, the writer focused on code switching used by English teacher at Eight Grade in Islam Tirtayasa Junior High School. In Qualitative inquiry uses a variety of procedures to confirm their research.

The writer were accumulated the data, described the result of the research and descriptive the data into data reduction, data display and conclusion drawing/verification.

\section{TECHNIQUE OF DATA ANALYSIS}

In analyzed the data, the writer focused on code switching used by English teacher at Eight Grade in Islam Tirtayasa Junior High School. In Qualitative inquiry uses a variety of procedures to confirm their research.

The writer were accumulated the data, described the result of the research and descriptive the data into data reduction, data display and conclusion drawing/verification.

\section{Data reduction}

Data reduction refers to the process of selecting, focusing, simplifying, abstracting and transforming the data that appears in written-up field notes transcriptions. To collect the data, the writer focused on observation in the online classroom activities by Zoom Apps to get the result. After observation, the writer identified the teacher about the reason uses code switching by interview.

\section{Data Display}


Universitas Banten Jaya

Data Display generally is an organized, compressed assembly of information that permits conclusion drawing and action. In this section, the writers begin analyzed and describe the data from teacher's conversation in online classroom activities by Zoom Apps to know the types of code switching and the reason in used code switching by the teacher at Eight Grade in Islam Tirtayasa Junior High School.

\section{Conclusion Drawing/Verification}

Conclusion drawing is only half of a Gemini configuration and also verified as an analyst proceeds. As we know that conclusion drawing and verification is the way to take summarize from observation and interview. In this step the writer use Mark and Casper formula are namely relative frequency. According Mark and Casper (2017, P.48) "relative frequency is equal in the frequency for an observed value of the data divided by the total number of data values is the sample. This formula is to get final result from type of code switching used by English teacher in number percentages.

The formula as follow;

$$
R F=\frac{f}{n} \times 100
$$

$$
\begin{aligned}
& \mathbf{R F}=\text { Relative Frequency } \\
& \mathbf{f} \quad=\text { Frequency }
\end{aligned}
$$

$\mathbf{n}=$ Total number of data values

\section{FINDING \& DISCUSSION}

The writer focused on the finding and discussion about code switching use by English teacher in eight grade at Islam Tirtayasa junior high school to identify the type of code switching use by English teacher in eight grade at Islam Tirtayasa junior high school and then to find out the reasons are code switching use by English teacher in eight grade at Islam Tirtayasa junior high school.

\section{A. Finding}

In this part the writer present the result from observation.

\section{Data Reduction}

The writer observed this research in two sections with Mrs. Septi Hartati as English teacher at Islam Tirtayasa Junior High School trough the teacher utterance in online classroom activities by Zoom Apps. The observation was started on $9^{\text {th }}$ until $16^{\text {th }}$ April 2020. The purpose of the research were to get data about the occurrence of code switching used by teacher at Eight Grade in Islam Tirtayasa Junior High School, to identify the types of code switching used by teacher and then to find out the reasons are code switching used by teacher when learning activities.

Before presenting the result of observation which discussed the types of 
Journal of English Language Teaching and Literature (JELTL)

Universitas Banten Jaya

code switching used by English teacher at eight grade in Islam Tirtayasa Junior High School and find out the reason are code switching used by English teacher at eight grade in Islam Tirtayasa Junior High School, the writer did data reduction. The writer sorted the data by only choosing utterance of conversation that occur on online classroom activities by Zoom Apps in two section and the data from observation was taken by photos and written transcript on appendices.

\section{Data Display}

The writer did data display in second step. In this step, the writer would display the result of transcript that has been simplified to make data to analyze. In the other hand, the result of transcribe would be identified to determine which were considered as code switching phenomenon as follow;

\section{A. The types of Code Switching used by English Teacher at Eight Grade in Islam Tirtyasa Junior High School}

\section{Table 4.2.1 The Result of Inter Sentential Switching}

\begin{tabular}{|c|c|c|}
\hline Observation & Teacher's Utterance & $\mathbf{C L}$ \\
\hline \multirow{13}{*}{$\begin{array}{c}\text { Observation } \\
1\end{array}$} & $\begin{array}{l}\text { Now, siapa yang belum joint in this } \\
\text { meeting? }\end{array}$ & CL 1.7 \\
\hline & And then, siapa lagi? & CL 1.9 \\
\hline & $\begin{array}{l}\text { Ok sambil menunggu mereka for joint this } \\
\text { meeting }\end{array}$ & CL 1.11 \\
\hline & $\begin{array}{l}\text { Still remember with the material yang miss } \\
\text { jelasin last meeting }\end{array}$ & CL 1.11 \\
\hline & That's true, jawabannya bener Dafin & CL 1.15 \\
\hline & $\begin{array}{l}\text { Okay, thank you Fatih, kita bisa mulai yah } \\
\text { anak-anak }\end{array}$ & CL 1.17 \\
\hline & $\begin{array}{l}\text { Ok open your book on pages.... Halaman } \\
\text { berapa tuh }\end{array}$ & CL 1.19 \\
\hline & $\begin{array}{l}\text { Okay, One Hundred Seventy Seven } \\
\text { tentang apa tuh? }\end{array}$ & CL 1.21 \\
\hline & $\begin{array}{l}\text { Okay, next pages lihat disitu yah dibagian } \\
\text { yang A yang look and say, sudah? }\end{array}$ & CL 1.23 \\
\hline & $\begin{array}{l}\text { So in part A kalian bisa lihat "look and } \\
\text { say" yang diawali dengan "Last Weekend" } \\
\text { selanjutnya dilanjutkan dengan kalimat } \\
\text { yang berhubungan dengan gambar }\end{array}$ & CL 1.25 \\
\hline & $\begin{array}{l}\text { So in part A you can see kalau gambar- } \\
\text { gambar tersebut bercerita tentang } \\
\text { kejadian di minggu lalu, kita sebut recount } \\
\text { text yakni menceritakan kejadian dimasa } \\
\text { lampau understand class? }\end{array}$ & CL 1.29 \\
\hline & $\begin{array}{l}\text { So look that percakapan antara Binsar } \\
\text { dan Atiqah }\end{array}$ & CL 1.31 \\
\hline & And the dialogue is bercerita tentang apa & CL 1.33 \\
\hline
\end{tabular}


Universitas Banten Jaya

\begin{tabular}{|c|c|}
\hline$h ?$ & \\
\hline $\begin{array}{l}\text { I mean is ya Atiqah and Binsar ngomongin } \\
\text { tentang apa Hisyam? }\end{array}$ & CL 1.35 \\
\hline $\begin{array}{l}\text { Where is Atiqah vocation last weekand, } \\
\text { ada yang tau? }\end{array}$ & CL 1.37 \\
\hline And then apalagi Dona? & CL 1.41 \\
\hline $\begin{array}{l}\text { Means of the water disitu untuk ombaknya } \\
\text { yah Dafin }\end{array}$ & CL 1.47 \\
\hline $\begin{array}{l}\text { It's mean bahwa kejadian ini terjadi } \\
\text { dimasa lampau atau sudah dilalu bukan } \\
\text { sedang berlangsung atau akan } \\
\text { berlangsung }\end{array}$ & CL. 1.51 \\
\hline $\begin{array}{l}\text { For now I want to give you all the exercise } \\
\text { pada bagian yang } \mathrm{C} \text {, sebelumnya ada } \\
\text { yang mau ditanyain ga? }\end{array}$ & CL 1.51 \\
\hline $\begin{array}{l}\text { Masih berkaitan dengan Atiqah and } \\
\text { Binsar in part B yang tadi }\end{array}$ & CL 1.53 \\
\hline $\begin{array}{l}\text { I give you fifty minutes, kalau ga paham } \\
\text { bisa ditanyakan, tinggal jawab true or } \\
\text { false and complete the text by Atiqah }\end{array}$ & CL 1.53 \\
\hline $\begin{array}{l}\text { Lanjut ke number two, true or false, bagus } \\
\text { coba jawab }\end{array}$ & CL 1.55 \\
\hline $\begin{array}{l}\text { That's true, and now giliran Radit buat } \\
\text { jawab number three true or false }\end{array}$ & CL 1.59 \\
\hline $\begin{array}{l}\text { And then number four giliran Decira yang } \\
\text { jawab true or false Decira }\end{array}$ & CL 1.61 \\
\hline $\begin{array}{l}\text { Giliran Abhi yang jawab number five true } \\
\text { or false Abhi please answer }\end{array}$ & CL 1.63 \\
\hline $\begin{array}{l}\text { Soalnya Atiqah bilang there weren't many } \\
\text { people there, disana tidak ramai orang }\end{array}$ & CL 1.67 \\
\hline $\begin{array}{l}\text { For the next true or false giliran siap nih, } \\
\text { ya udah coba Navra answer for number } \\
\text { six true or false? }\end{array}$ & CL 1.67 \\
\hline $\begin{array}{l}\text { Ya, jawabannya False, karena disitu } \\
\text { dijelasin kalau the water was calm yah } \\
\text { anak-anak, next true or false anak-anak }\end{array}$ & CL 1.69 \\
\hline $\begin{array}{l}\text { The answer is true karena Atiqah } \\
\text { menyarankan Binsar untuk pergi kesana }\end{array}$ & CL 1.73 \\
\hline $\begin{array}{l}\text { For number one sudah ada jawabannya, } \\
\text { so next on number two sekarang Zickrina } \\
\text { yang jawab }\end{array}$ & CL 1.73 \\
\hline $\begin{array}{l}\text { Yes true the answer is sand, and then for } \\
\text { the next number jawabannya apa anak- } \\
\text { anak }\end{array}$ & CL 1.81 \\
\hline And the last jawabannya apa? & CL 1.85 \\
\hline $\begin{array}{l}\text { Ok finish class buat latihannya yang part } \\
\mathrm{D} \text { and } \mathrm{E} \text {, any question? }\end{array}$ & CL 1.87 \\
\hline $\begin{array}{l}\text { And for your homework tulis tentang } \\
\text { kejadian dimasa lampau kalian baik itu }\end{array}$ & CL 1.89 \\
\hline
\end{tabular}


Universitas Banten Jaya

\begin{tabular}{|c|c|c|}
\hline & $\begin{array}{l}\text { last week last month or yesterday minimal } \\
\text { lima kalimat }\end{array}$ & \\
\hline \multirow{26}{*}{$\begin{array}{l}\text { Observation } \\
2\end{array}$} & $\begin{array}{l}\text { Baiklah hari ini kita kembali belajar mata } \\
\text { pelajaran Bahasa Inggris yah anak-anak, } \\
\text { do you have a homework? }\end{array}$ & CL 2.7 \\
\hline & $\begin{array}{l}\text { Okay class siapa nih yang belum joint this } \\
\text { meeting sekarang? }\end{array}$ & CL 2.14 \\
\hline & $\begin{array}{l}\text { Baiklah sambil menunggu miss mau tanya, } \\
\text { still remember about what we have study } \\
\text { last week? }\end{array}$ & CL 2.19 \\
\hline & About what? Tentang apa? & CL 2.21 \\
\hline & Ya that's true, and then apa lagi? & CL 2.23 \\
\hline & How the weather? Cuacanya gimana? & CL 2.31 \\
\hline & And dengan siapa Atiqah goes to beach? & CL 2.35 \\
\hline & $\begin{array}{l}\text { Pada percakapan tersebut with whom } \\
\text { Atiqah was talking? }\end{array}$ & CL 2.37 \\
\hline & $\begin{array}{l}\text { Okay now open on pages } 182, \underline{b u k a} \\
\text { halaman } 182\end{array}$ & CL 2.47 \\
\hline & $\begin{array}{l}\text { Kalimat dibawahnya menjelaskan about } \\
\text { the activity }\end{array}$ & CL 2.47 \\
\hline & And the meaning is? Artinya apa Nazla & CL 2.51 \\
\hline & $\begin{array}{l}\text { And for the next please Mozaweka coba } \\
\text { bacakan kalimatnya }\end{array}$ & CL 2.53 \\
\hline & $\begin{array}{l}\text { And then Rangga Unggu turn buat bacain } \\
\text { kalimat selanjutnya silahkan Rangga }\end{array}$ & CL 2.57 \\
\hline & $\begin{array}{l}\text { Next Khusnul turn for read aloud kalimat } \\
\text { selanjutnya berserta artinya yah }\end{array}$ & CL 2.61 \\
\hline & And the last apa anak-anak & CL 2.65 \\
\hline & Just kidding yah anak-anak & CL 2.67 \\
\hline & $\begin{array}{l}\text { Miss kasih waktu kalian } 10 \text { menit yah and } \\
\text { then we are move to next question, do you } \\
\text { understand? }\end{array}$ & CL 2.70 \\
\hline & Finish class? Sudah selesai anak-anak? & CL 2.72 \\
\hline & $\begin{array}{l}\text { Okay for number one, who wrote the } \\
\text { email, siapa yang menulis emailnya? }\end{array}$ & CL 2.74 \\
\hline & $\begin{array}{l}\text { Yeah that true and for number two } \\
\text { jawabannya adalah, Rizki coba jawab }\end{array}$ & CL 2.76 \\
\hline & $\begin{array}{l}\text { And then for number three is sekarang } \\
\text { giliran Dona, coba ayo jawab }\end{array}$ & CL 2.78 \\
\hline & $\begin{array}{l}\text { Next number, what she does on the first } \\
\text { day of her vacation? Dafin silahkan jawab }\end{array}$ & CL 2.82 \\
\hline & $\begin{array}{l}\text { And then for the next question apa nih } \\
\text { jawabannya anak-anak }\end{array}$ & CL 2.84 \\
\hline & $\begin{array}{l}\text { For the next number apa nih jawabannya, } \\
\text { what did she buy there Muhtado please }\end{array}$ & CL 2.86 \\
\hline & $\begin{array}{l}\text { The last question did she have a good time } \\
\text { in the shops? Siapa yang bisa jawab? }\end{array}$ & CL 2.88 \\
\hline & Okay finish buat latihannya, dan yang tadi & CL 2.92 \\
\hline
\end{tabular}


Universitas Banten Jaya

\begin{tabular}{|l|l|l|} 
& $\begin{array}{l}\text { about email from Lidia to Johan is } \\
\text { example of Recount text dalam bentuk } \\
\text { pesan yah anak-anak, sampe sini ada yang } \\
\text { mau ditanyakan ga? }\end{array}$ & \\
\cline { 2 - 3 } & $\begin{array}{l}\text { Okay before we ended this study miss } \\
\text { punya pekerjaan rumah lagi buat kalian }\end{array}$ & CL 2.96 \\
\cline { 2 - 3 } & $\begin{array}{l}\text { Open pages on 187, look at part A disitu } \\
\text { sama kaya yang sebelumnya tapi } \\
\text { bentuknya text cerita yah anak-anak }\end{array}$ & CL 2.96 \\
\cline { 2 - 3 } & $\begin{array}{l}\text { Find the verb2 at this text yah anak-anak, } \\
\text { do you remember? }\end{array}$ & CL 2.96 \\
\cline { 2 - 3 } & $\begin{array}{l}\text { Okay class thank you for today dan terima } \\
\text { kasih buat perhatiannya }\end{array}$ & CL 2.98 \\
\hline $\begin{array}{l}\text { Total Inter } \\
\text { Sentential } \\
\text { Switching }\end{array}$ & \multicolumn{1}{|c}{$\mathbf{6 4}$} & \\
\hline
\end{tabular}

2. Table 4.2.2 The Result of Intra Sentential Switching

\begin{tabular}{|c|c|c|}
\hline Observation & Teacher's Utterance & $\mathbf{C L}$ \\
\hline \multirow{14}{*}{$\begin{array}{c}\text { Observation } \\
1\end{array}$} & $\begin{array}{l}\text { So yang lain mengertikan dengan gambar } \\
\text { gambar teresbut }\end{array}$ & CL 1.27 \\
\hline & $\begin{array}{l}\text { Sekarang next ke bagian B "Listen to the } \\
\text { dialogue" }\end{array}$ & CL 1.31 \\
\hline & Now giliran Nayla Gina & CL 1.43 \\
\hline & $\begin{array}{l}\text { So yang dimkasud dari "the water was } \\
\text { calm" adalah bahwa ombaknya tenang yah } \\
\text { anak-anak }\end{array}$ & CL 1.47 \\
\hline & Simak baik-baik yah class & CL 1.49 \\
\hline & $\begin{array}{l}\text { Recount Text menggunakan kalimat past } \\
\text { tense }\end{array}$ & CL 1.49 \\
\hline & $\begin{array}{l}\text { Jadi verbnya menggunakan verb kedua yah } \\
\text { anak-anak... kalo present tense kan pake } \\
\underline{\text { verb } 1 \text { yah kalo past tense pake verb }} \\
\text { kedua, sampe sini faham? }\end{array}$ & CL 1.49 \\
\hline & $\begin{array}{l}\text { Penggunaan verb kedua pada percakapan } \\
\text { diatas }\end{array}$ & CL 1.51 \\
\hline & Okay and the last jawabannya true or false & CL 1.71 \\
\hline & $\begin{array}{l}\text { Finish for part D and then kita move on to } \\
\text { part E }\end{array}$ & CL 1.73 \\
\hline & Iya air udara yah bukan water & CL 1.75 \\
\hline & $\begin{array}{l}\text { Next Bakhtiar jawabannya apa buat nomor } \\
\text { 3, silakan dijawab }\end{array}$ & CL 1.75 \\
\hline & $\begin{array}{l}\text { Okay siapa yang bisa answer pertanyaan } \\
\text { nomor } 3\end{array}$ & CL 1.77 \\
\hline & $\begin{array}{l}\text { For number four is zidane turn, silakan } \\
\text { answer Zidane }\end{array}$ & CL 1.79 \\
\hline
\end{tabular}


Universitas Banten Jaya

\begin{tabular}{|c|c|c|}
\hline & $\begin{array}{l}\text { selanjutnya answer for question of number } \\
\text { six }\end{array}$ & CL 1.83 \\
\hline & $\begin{array}{l}\text { Baiklah untuk pertemuan selanjutnya kita } \\
\text { ke part dua tentang "we walked to the } \\
\text { beach" }\end{array}$ & CL 1.89 \\
\hline & $\begin{array}{l}\text { dikumpulkan paling lambat sehari sebelum } \\
\text { kelas kita dimulai, bisa kirim by WA ke } \\
\text { miss aja nanti ditulis dibuku lalu difotokan } \\
\text { dam kirim ke miss disertai nama yah }\end{array}$ & CL 1.89 \\
\hline \multirow{14}{*}{$\begin{array}{c}\text { Observation } \\
2\end{array}$} & $\begin{array}{l}\text { Siapa yang belum mengumpulkan } \\
\text { homeworknya ke miss kemarin? }\end{array}$ & CL 2.9 \\
\hline & Who yang goes to the beach? & CL 2.27 \\
\hline & The names of the beachnya apa? & CL 2.29 \\
\hline & $\begin{array}{l}\text { Was it crowded at the beach? Beachnya } \\
\text { ramai? }\end{array}$ & CL 2.33 \\
\hline & That's good kids kalian cool & CL 2.39 \\
\hline & $\begin{array}{l}\text { See pada bagian } 2 \text { yah yang "we walked to } \\
\text { the beach" hampir sama kaya bagian } 1\end{array}$ & CL 2.47 \\
\hline & Disitu you can see the picture & CL 2.47 \\
\hline & First silakan baca Nazla & CL 2.49 \\
\hline & Iya that's true Unggu & CL 2.61 \\
\hline & And then Haekal turns, silakan Haekal & CL 2.63 \\
\hline & Udah udah stop kita lanjut ke part B yah & CL 2.70 \\
\hline & Good Halim ya betul & CL 2.82 \\
\hline & $\begin{array}{l}\text { So jadi recount text itu bercerita tentang } \\
\text { kondisi, situasi, aktivitas atau peristiwa } \\
\text { dimasa lampau yah anak-anak }\end{array}$ & CL 2.96 \\
\hline & $\begin{array}{l}\text { So yang tidak ikut hari ini miss anggep ha } \\
\text { hadir yah }\end{array}$ & CL 2.98 \\
\hline $\begin{array}{l}\text { Total of } \\
\text { Intra- } \\
\text { sentential } \\
\text { Code } \\
\text { Switching }\end{array}$ & \multicolumn{2}{|l|}{ e } \\
\hline
\end{tabular}

3. Table 4.2.3 The Result of Tag Switching

\begin{tabular}{|c|c|c|}
\hline Observation & Teacher Utterance & $\mathbf{C L}$ \\
\hline \multirow{4}{*}{$\begin{array}{c}\text { Observation } \\
1\end{array}$} & $\underline{N a h}$, still remember about recount text? & CL 1.13 \\
\hline & Yaps the answer is true & CL 1.61 \\
\hline & Let's say hamdalah & CL 1.91 \\
\hline & $\begin{array}{l}\text { Alhamdulillah See you next meeting } \\
\text { Wassalamualaikum warrahmatullahi } \\
\text { wabarrakatuh } \\
\end{array}$ & CL 1.91 \\
\hline Observation & We can start now yah & CL 2.41 \\
\hline
\end{tabular}


Universitas Banten Jaya

\begin{tabular}{|c|l|c|}
\multicolumn{1}{|c|}{2} & $\begin{array}{l}\text { Nah, in part B there is an email from Lidia } \\
\text { to Johan }\end{array}$ & CL 2.70 \\
\cline { 2 - 3 } & $\begin{array}{l}\text { Yaps, good answer Dafin } \\
\text { Aha good Dafin, the answer is she walked } \\
\text { to the beach }\end{array}$ & CL 2.92 1.84 \\
\cline { 2 - 3 } & Let's say hamdalah & CL 2.98 \\
\cline { 2 - 3 } & $\begin{array}{l}\text { See you next time, Assalamualaikum } \\
\text { warrahmatullahi wabarrakatuh }\end{array}$ & CL 2.100 \\
\hline \multirow{2}{*}{$\begin{array}{l}\text { Total Tag } \\
\text { Switching }\end{array}$} & $\mathbf{1 0}$ & \\
\hline
\end{tabular}

3. Conclusion Drawing / Verification

In the last step are Conclusion

Drawing / Verification. The writer gave the conclusion of the result from the data

a. The Percentages Types of Code Switching Used by English Teacher at Eight Grade in Islam Tirtayasa Junior High School

Table 4.2.4

\begin{tabular}{|c|c|c|c|}
\hline No & $\begin{array}{c}\text { Types of Code Switching } \\
\text { That Occur on Learning } \\
\text { Process in Eight Grade } \\
\text { at Islam Tirtayasa } \\
\text { Junior High School }\end{array}$ & Frequency & Percentages (\%) \\
\hline 1 & Inter Sentential Switching & 64 & $60,95 \%$ \\
\hline 2 & Intra Sentential Switching & 31 & $29,52 \%$ \\
\hline 3 & Tag Switching & 10 & $9,52 \%$ \\
\hline \multicolumn{2}{|c|}{ Total } & $\mathbf{1 0 5}$ & $\mathbf{1 0 0 \%}$ \\
\hline
\end{tabular}

b. Number Percentages of Code Switching

1) Inter Sentential Switching

$$
\mathrm{RF}=\frac{64}{105} \times 100=60,95 \%
$$

2) Intra Sentential Switching

$$
\mathrm{RF}=\frac{31}{105} \times 100=29,52 \%
$$

3) Tag Switching reduction and data display to verify analyst proceeds using Mark and Casper formula. The result will be discussed below; 
Universitas Banten Jaya

$$
\mathrm{RF}=\frac{10}{105} \times 100=9,52 \%
$$

c. Chart result of Types of Code Switching Used by English Teacher in Eight Grade at Islam Tirtayasa Junior High School

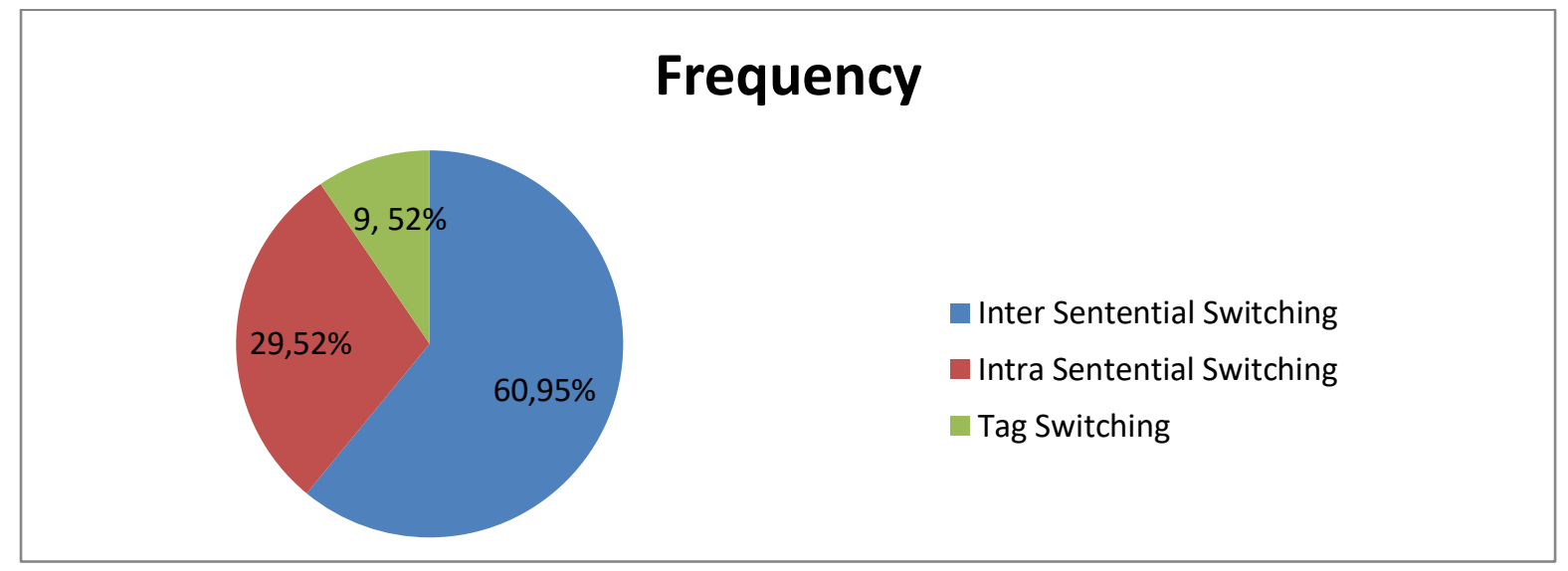

d. The Reason of Code Swithcing That Used by English Teacher in Eght Grade at Islam Tirtyasa Junior High School

In this part the writer showed of interview from English teacher in online classroom activities at Islam Tirtyasa Junior High School. The result will be discussed below;

In the first question the writer asked about the reason code switching used when the teacher teaches in online classroom activities and English teacher answered that;

"Alasannya karena mayoritas, tidak mayoritas sih tapi beberapa anak belum mengerti banget bahasa inggris jadi untuk mempermudah KBM jadi kita menggunakan code swithcing" (CL 3.8). You can see on Appendices.

In the second question is the writer asked about what are the goals of code switching used in online classroom activities. Then the English teacher "Tujuannya supaya mereka lebih paham tentang materi yang kita berikan, jadi supaya mempermudahkan saja, jadi ketika anak itu bingung kita pertama menjelaskan bahasa inggris setelah itu kan biasanya mengerti gak tuh, ada yang beberapa mengerti dan ada yang engga, ada beberapa yang mengerti itu enak kan yah gampang dan kalau gak ngerti tuh biasanya kita ngasih kuis ke bahasa indonesia nah itu baru mereka paham memudahkan"answered;

(CL 3.10). You can see on Appendices.

In the third question, the writer asked about the important code switching used in online classroom activities. Then the English teacher answered;

"Sebenernya sih ya penting gak penting ya, tapi karena kita kan mayoritas orang indonesia banget kan dan bahasa inggris 
Universitas Banten Jaya

sebagai foreign language bukan second language jadi dimana bahasa inggris itu sebagai bahasa asing, mereka umumnya belum mengerti bahasa inggris ya mungkin merekapun cuma ngomong-ngomong bahasa inggris gitu aja kan dilagu tapi mereka tuh gak tau artinya tuh apa, nah sebenernya itu penting untuk pembelajaran karena memudahkan KBM"'(CL 3.13). You can see on Appendices.

From each reason the writer can conclude that the reason of using code switching that used by English teacher such as;

1. Talking about particular topic

People sometimes prefer to talk about a particular topic in one language.

\section{Interjection}

Interjection is words or expressions which are inserted into a sentence to convey surprise, strong emotion that explains by Hoffman. Therefore the used interjection is as connector in their language.

\section{Repetition for clarification}

According to Hoffman repetition is not only served on clarify what is said, but also to amplify or emphasize a message. Therefore, the teacher used repetition in order the student can understand what the teacher utterances.

\section{Expressing group identity}

The last reason are to expressing group identity, sometime someone who speaks foreign language to expressing group identity in because they want to expressing some group identity.

From four reasons above was used by English teacher in online classroom activities where are the data based on the interview with English teacher at Eight Grade in Islam Tirtayasa Junior High School.

\section{B. Discussion}

1. The Types of Code Switching That Used by English Teacher in Eight Grade at Islam Tirtyasa Junior High School

\section{a. Inter-Sentential Code Switching}

The first types of code switching that used by English Teacher in Eight Grade at Islam Tirtayasa Junior High School are Inter sentential code switching. According to Holmes (2013, P. 44-45) "Inter sentential code switching is people who are less proficient will tend to switch at sentence boundaries". In the other hand, each teacher has purpose in using these types for English teacher has one aim in orders the student become easier to understand when the teacher gives instruction. But make English teacher has purpose to the student more easily in understanding the information. For example 
Universitas Banten Jaya

of Inter sentential code switching for each teacher below;

Teacher: I give you fifty minutes, kalau ga paham bisa ditanyakan, tinggal jawab true or false and complete the text by Atiqaacher: Okay class thank you for attention and for (CL 1.53).

From the example above, the teacher switch their code to make an instruction and give information that comprehensible for the student.

\section{b. Intra-Sentential Code Switching}

This type occur within in a sentences, as define by Jendra (2010, p.7577) "this type found when a word, a phrase, a clause of a foreign language is found within the sentences in a base language". In this part, teacher purpose is to give an assist for the students to understand the meaning of new vocabularies.

For example that mention below;

Teacher: Iya air udara yah bukan water (CL $1.75)$.

From the example above, the teacher switch their code to gives assist for the students to understand the meaning of new vocabularies and also to clarify their utterances

\section{c. Tag Switching}

As define by Holmes (2013, p. 4445) "Tag switching is simply an interjection or a linguistic tag in the other language which serves as an ethnic/culture identity marker". The teacher purpose in using tag switching is to convey short expression which related to language culture and to convey a habitual for praying. For example that mention by each teacher below joint in this meeting, let say hamdalah (CL 1.91).

Teacher: Nah, in part B there is an email from Lidia to Johan (CL 2.70).

From the example above, the teacher used their code based on their mother tongue like, nah, yaps, or insert short expression. Besides that the teacher used culture of language to said greeting in the classroom.

\section{The Types of Code Switching That Unused English Teacher in Eight Grade at Islam Tirtayasa Junior High School}

In this part, the writer discuss about types of code switching unused by English teacher in Eight Grade at Islam Tirtayasa Junior High School.

Based on the observation, the types that unused by English teacher in Eight Grade in Islam Tirtayasa Junior High School are situation code switching and metaphorical code switching.

According to Wardhaugh (2015, p.98) "Situation code switching occurs when the languages used change according to the situations in which the conversant find themselves". Therefore, this type can't manipulate in the classroom activities 
Universitas Banten Jaya

because the situation not changed. This classroom activities use online classroom activities by Zoom Apps. The next code switching that unused by English teacher in Eight Grade at Islam Tirtayasa Junior High School are metaphorical code switching.

According to Jendra (2010, P.75-77)

"This type happens when there is a change in the perceptions or the purpose, or the topic of the conversations". Based on the observation, the teacher does not used code switching in their perception or opinion. Therefore, that type unused by teacher in Eight Grade in Islam Tirtyasa Junior High School.

From the explanation above, the writer concluded that types code switching used by English teacher in Eight Grade at Islam Tirtayasa Junior High School are inter sentential code switching, intra sentential code switching and tag switching. Moreover, the types of code switching that unused by English teacher in Eight Grade at Islam Tirtayasa Junior High School are situational code switching and metaphorical code switching.

\section{The Reason of Using Code Switching}

That Used by English Teacher in Eight Grade at Islam Tirtayasa Junior High School

\section{a. Talking About Particular Topic}

People sometimes prefer to talk about a particular topic in one language.
This reason came from English teacher. The English teacher feels comfort to switch their codes about particular topic. For example from English teacher;

Teacher: It's mean bahwa kejadian ini terjadi dimasa lampau atau sudah dilalu bukan sedang berlangsung atau akan berlangsung

In the sample above, the English teacher teaching recount text and the teacher explain about the meaning of recount text. In this case the teacher used code switching in order when the teacher explains about the topic the students can be understand.

\section{b. Interjection (inserting sentence fillers or sentence connectors)}

Interjection is words or expressions which are inserted into a sentence to convey surprise, strong emotion that explains by Hoffman. Therefore the used interjection is as connector in their language. In the other hand, the English teacher used interjection in a sentence to indicate that the teacher wanted to attention from the student. For example from the English teacher that mention below;

Teacher: Udah udah stop kita lanjut ke part B yah

In the example above, the teacher gives strong emotions to students so that students can calm down and not be rowdy and continue learning poses. 
Universitas Banten Jaya

\section{c. Repetition for Clarification}

According to Hoffman repetition is not served to clarify what is said, but also to amplify or emphasize a message. Therefore, the teacher used repetition in order the students can understand what the teacher utterance but sometime the teacher used repetition in another language. In this case came from English teacher. For Example that mention by teacher below;

Teacher: How the weather? Cuacanya gimana?

In the teachers' utterances above, teacher used code switching at the previous sentences in order the students can be understand at the English sentences that repeat into Indonesia language.

\section{d. Expressing Group Identity}

According to Hoffman expressing group identity in code switching is the ways people communicate with their group are obviously different from the way they communicate with other people from other groups. When the writer interviewed the English teacher, she said about majority of Indonesian people language. That mention below;

Teacher: tapi karena kita kan mayoritas orang indonesia banget kan dan bahasa Inggris sebagai foreign language bukan second language jadi dimana b.inggris itu sebagai bahasa asing
In this case, the teacher want to explain about English is foreign language for Indonesian people not second language.

\section{The Reason of Using Code Switching That Unused by English Teacher in Eight Grade at Islam Tirtayasa Junior High School}

In this part, the writer will discuss about the reason unused English teacher in Eight Grade at Islam Tirtayasa Junior High School.

Based on the interview, the reason that unused by English teacher in Eight Grade at Islam Tirtayasa Junior High School are Quoting somebody else, being emphatic about something, and Intention of clarifying the speech content for interlocutor. All of them can't manipulate because the English teacher was convery that doing code switching should be based on what our needed in classroom activities.

Based on the explanation above, the writer can concluded that their reason in using code whitching from 7 reasons based on Hoffman and Jendra theory from each teacher based on gender inequality only used 4 reasons. The reason are talking about particular topic, repetition for clarification, interjection and expressing group identity. Therefore, there are 3 reasons that unused by English teacher 
Universitas Banten Jaya

based on gender inequality ore quoting somebody else, being emphatic about something, intention of clarifying the speech content for interlocutor.

\section{CONCLUSION}

Based on data analysis the writer can conclude that:

1. The types of code switching that used by English teacher in Eight Grade at Islam Tirtayasa Junior High School in online classroom activities were mainly three types. Where are the types of code switching proposed by Holmes and Jendra theory, these types were: 1). Inter sentential code switching; 2). Intra sentential code switching; 3). Tag switching. In the other hand, each types that used by English teacher in eight grade at Islam Tirtayasa Junior High School was made in number percentages are inter sentential code switching $60,95 \%$ intra sentential code switching 29,52\% and tag switching $9,52 \%$.

2. The reason of English teacher in Eight Grade at Islam Tirtayasa Junior High School that used code switching in classroom activities were mainly four reasons. These reason were; 1). Talking about particular topic; 2). Interjection (inserting sentence fillers or sentence connectors); 3). Repetition for clarification; 4). Expressing group identity.It summarizes to your new findings based on the research. It may state the strengths and weaknesses of your methodology. It may also specify the room for future improvement.

\section{REFERENCES}

Baker, Colin. 2011. Foundation of

Bilingual Educations and

Bilingualism. US of America:

Longman.

Bassiouney7T2,R \& 1T2Katz 1T7E. G (2012) 7T 2T Arabic Language and Linguistics, Georgetown: University Press.USA..

Bogdan, Robert C \& Biklen. Sari Knoop. 2007. Qualitative Research for

Education. An Introduction to

Theory and Method, fifth edition. ISBN 0-205-07695-5.

Creswell, John W. 2014. Research Design: Qualitative, Quantitative and Mixed Method Approach: Fourth Edition. London. Sage Publications Ltd

Dewi, Citra \& Yayan Ekalaya. 2015. An Analysis of Outer Code Switching and Code Mixing in Indonesia Lawyers Club. Dehasen University. Hamid, Siti Maryam. 2016. Code Switching Between the Teacher and the Student of the Conversation. 
Universitas Banten Jaya

Exposure Journal, Vol. 5 No. 2

November 2016, P.174-193.

Heredia, Roberto Ramirez \& Jeanette

Altarriba. 2018. Bilingual Language

Mixing: Why Do Bilinguals Code-

Switch?. Vol. 10, No. 5, October

2001, P.164-172.

Holmes, Janet. 2013. An Introduction to Sociolinguistics, Fourth Edition.

New York: Longman.

Hudson, R.A. 2012. An Introduction to

Sociolinguistics: Second Edition.

London: Cambridge

University Press.

Jendra, Iwan Indrawan. 2010.

Sociolinguistics: The Study of

Societies' Language. Yogyakarta:

Graha Ilmu Publishing.

Jourdan, Christine and Kevin Tuite. 2006.

Language, Culture, and Society.

Cambridge: Cambridge University

Press.

Kothari, C. R. 2014. Research Methodology

Method and Technique: Third

Revised Edition. New Age

International (P) Ltd.

Mack. Natasha. Woodsong. Chyntia. 2005.

Qualitative Research Methods: a data Collector's Field Guide.

U.S. Agency fot International

Development (USAID)

Marczyk, Geoffrey. David Dematteo and

David Festinger. 2005. Essential of
Research Design Methodology. New Jersey: Jhon Wiley and Sons, Inc.

Mark, Beintema and Natalia Casper. 2017. Introductory Statistics. College of Lake County Faculty.

Mesthrie, Rajed. Joan Swann. Anna Deumert and William L. Leap. 2009. Introducing Sociolinguistics. Edinburgh University Press.

Mujiono, Soepomo Poedjosoedarmo, Edi Subroto and Tri Wiranto. "Code Switching in English as Foreign Language Instruction Practiced by the English Lecturers at Universities". Vol : 5.2 (2013) : 5051. Print.

Miles, B. Matthew. Hubberman Michael and Sadana, J. (2014). Qualitative Data Analysis, A. Methods Sourcebook, Third Edition. USA: Sage Publications

Qonaatun, Aa. "The Usage Factors of Code Switching In Teaching Interaction of English Class". Vol. 1 No. 1 (2018). P. 18-35.

Sugiyono, 2008. Metode Penelitian Kuantitatif, Kualitatif dan $R \& D$. Bandung: Alfabeta

Trousdale, Graeme. 2010. An Introduction to English Sociolinguistics. Edinburgh University Press Ltd. 
Journal of English Language Teaching and Literature (JELTL)

Volume 4 No. 1, February 2021

E-ISSN 2622-9056

Universitas Banten Jaya

Wardhaugh, Ronald and Janet M. Fuller.

2015. An Introduction to

Sociolinguistics: $\quad$ Seventh

Edition. USA: Backwell

Publishing.https://owlcation.com/humanitie

s/Code-Switching-Definition-

Types-and-Examples-of-Code-

Switching. 
Journal of English Language Teaching and Literature (JELTL)

P-ISSN 2623-0062

Volume 4 No. 1, February 2021

E-ISSN 2622-9056

Universitas Banten Jaya 